\title{
Blastocoele expansion: an important parameter for predicting clinical success pregnancy after frozen-warmed blastocysts transfer
}

\author{
Jing Zhao ${ }^{1,2}$, Yi Yan$^{1}, \mathrm{Xi} \mathrm{Huang}^{1}$, Lunquan Sun ${ }^{2}$ and Yanping Li ${ }^{1 *}$
}

\begin{abstract}
Objective: To assess the predictive value of each individual morphological parameter: blastocoele expansion degree, inner cell mass (ICM), and trophectoderm (TE) grades on the clinical pregnancy outcome in frozen-warmed embryo transfer (FET) cycles.

Methods: This is a retrospective cohort study, including 1154 FET cycles receiving vitrified-warmed one or two blastocysts transfer from August 2011 through to May 2018. The correlation between blastocyst morphology parameters and clinical outcome after FET was assessed.

Results: In the subgroup analysis based on clinical pregnancy, the patients who achieved clinical pregnancy had a significantly higher degree of blastocyst expansion ( $3.69 \pm 0.68 \mathrm{vs} .3 .53 \pm 0.78, P=0.000)$ and had a thicker endometrium ( $9.65 \pm 1.63$ vs. $9.28 \pm 1.64)$ compared with those with non-clinical pregnancy. The logistic regression analysis showed that among the three blastocyst morphology parameters, only the blastocoele expansion degree was significantly correlated with the clinical pregnancy outcome and had ability to predict the outcome after FET cycles with one or two vitrified-warmed blastocysts transferred. Both ICM and TE stages were not associated with pregnancy outcomes.
\end{abstract}

Conclusions: The blastocoele expansion degree may be essential for successful pregnancy and should be given priority when selecting frozen blastocyst for transfer.

Keywords: Blastocoele expansion, Inner cell mass, Trophectoderm, Blastocyst morphology, Frozen-warmed embryo transfer

\section{Introduction}

With the successful introduction of embryo vitrification, vitrified-warmed embryo transfer cycles, which preserve excess embryos derived from IVF or ICSI treatment cycles and transfer later, are a vital part of assisted reproductive technology (ART). Compared with cleavage embryo, blastocyst culture as a tool for selecting embryo has improved clinical pregnancy outcomes [1-3]. Besides, blastocyst transfer was regarded to enhance the synchrony between embryonic development and endometrium [4].

\footnotetext{
* Correspondence: LYP7798@126.com

${ }^{1}$ Reproductive Medicine Center, Xiangya Hospital, Central South University, 87 Xiangya Road, Changsha City, Hunan Province, People's Republic of China Full list of author information is available at the end of the article
}

So, in the clinical practice, blastocyst vitrification and frozen - warmed embryo transfer (FET) in the following cycles is suggested when there have surplus embryos.

Unlike the intricate ovarian stimulation protocols used to stimulate multiple follicular developments, the FET protocols are simpler, and its purpose is limited to adequate preparation of the endometrium and selection good-quality embryos/blastocysts. In 2013, one metaanalysis showed that all current endometrial preparation methods appear to be equally successful in terms of ongoing pregnancy rate [5]. Therefore, selecting goodquality embryos with optimal implantation potential is critical to ensure that each infertile couple has the maximum likelihood of getting pregnant after ART.

(c) The Author(s). 2019 Open Access This article is distributed under the terms of the Creative Commons Attribution 4.0 International License (http://creativecommons.org/licenses/by/4.0/), which permits unrestricted use, distribution, and reproduction in any medium, provided you give appropriate credit to the original author(s) and the source, provide a link to the Creative Commons license, and indicate if changes were made. The Creative Commons Public Domain Dedication waiver (http://creativecommons.org/publicdomain/zero/1.0/) applies to the data made available in this article, unless otherwise stated. 
However, there is poorly consensus on the blastocyst selection.

At present, morphological evaluation has been widely used to predict embryo vitality as a relatively simple and non-invasive method. Several blastocyst-grading systems have been proposed to assess blastocyst quality. The blastocyst grading system proposed by Gardner and Schoolcraft [6] was based on blastocyst morphology parameters and was kept basically unchanged. Some studies have reported that trophectoderm (TE) grade has a stronger predictive power than the ICM grade and the blastocyst expansion degree in estimation of outcome after blastocyst transfer [7-10] while some studies showed that the ICM grade was more important [11, 12]. However, some researches indicated that the clinical outcomes after single blastocyst transfer could be predicted by blastocoele expansion degree $[9,13,14]$. And recently, Sun YP firstly confirmed that the blastocoele expansion and re-expansion degree had a stronger ability to predict live birth than ICM or TE grade in fresh and vitrified/ warmed single blastocyst transfer cycles [5].

This relationship among each parameter of the blastocyst morphology and outcomes of ART is inconsistent and is not fully understood. In addition, as far as we know, the correlation between blastocyst parameters and outcomes after transferring non-selective single or two blastocysts in FET cycles has not been examined.

So, the aim of the present study was to assess the prognostic value of each individual morphological parameter (blastocoele expansion degree, ICM, and TE grade) on the clinical pregnancy outcome in FET cycles.

\section{Materials and methods}

\section{Study design}

This study was approved by the Ethical Committee of Xiangya Hospital, Central South University, and was performed in the light of the Declaration of Helsinki. This study retrospectively analyzed infertile couples who received frozen-warmed blastocyst transfer at the Reproductive Medical Center of the Xiangya Hospital from August 2011 through to May 2018. The inclusion criteria: 1) frozen-warmed blastocyst transfer cycles with one or two blastocysts transferred; 2) for women who received two blastocysts transferred, cycles in which non or two blastocysts implanted were included. The main exclusion criteria: 1) preimplantation genetic diagnosis/ screening (PGD/PGS) cycles; 2) women with intrauterine adhesion (IUA); 3) fresh blastocyst transfer cycles.

\section{Ovarian stimulation protocol}

All the vitrified/warmed blastocysts transferred during the later cycles were acquired during ovarian stimulation cycles as described previously [15-18]. The personalized individual ovarian stimulation protocol was given basing on the age, infertility factor, $\mathrm{AMH}$, and antral follicular count (AFC). When the serum estrogen $<50 \mathrm{pg} / \mathrm{ml}$, and diameter of the largest follicle $<10 \mathrm{~mm}$, ovarian stimulation initiated. One hundred fifty to Three hundred IU recombinant FSH and/or human menopausal gonadotrophin were used to promote follicle development. Follicle was triggered with 5000-10,000 IU of hCG when at least two follicles $>18 \mathrm{~mm}$. Oocytes were picked up $36 \mathrm{~h}$ after hCG injection, and IVF or ICSI was conducted according to the practice protocol.

\section{Blastocyst vitrification and warming}

If there were surplus embryos after transfer in fresh cycles, these embryos were suggested to be cultured to form blastocyst. According to the methods reported by Lane et al. [19] and Mukaida et al. [20, 21], blastocysts were vitrified and warmed. Embryologists artificially shrink the blastocysts with a laser pulse prior to vitrification. Blastocysts were firstly transferred to an equilibration solution composed of 7.5\% DMSO and 7.5\% ethyleneglycol for 8-10 min, and then were transferred to a vitrification solution containing 15\% DMSO +15\% ethylene glycol $+0.5 \mathrm{M}$ sucrose for $1 \mathrm{~min}$. Subsequently, each blastocyst was placed into a cryoloop, plunged into liquid nitrogen, and then covered with a cryotube and stored in liquid nitrogen.

To warm, the blastocyst loaded in the cryoloop was immersed in the first thawing solution with $1.0 \mathrm{M}$ sucrose at room temperature for $2 \mathrm{~min}$, and then transferred to the second thawing solution containing $0.5 \mathrm{M}$ sucrose for $3 \mathrm{~min}$. At last, blastocysts were washed in the wash solution, at $37^{\circ} \mathrm{C}$ for $10 \mathrm{~min}$. After warming up, the blastocysts were cultured in culture medium for $3 \mathrm{~h}$.

In light of Gardner and Schoolcraft's blastocyst grading system, vitrified/ warmed blastocysts were graded $2 \mathrm{~h}$ after thawing of blastocyst [6]. Blastocysts were graded by two embryologists who have more the 5 years of work experience, aiming to decrease subjective errors. Blastocysts with $>3 \mathrm{BB}$ grade were deemed to be viable and were transferred.

\section{Frozen-warmed blastocyst transfer cycle}

In the natural cycle FET (NC-FET), we monitored the development of the dominant follicle and endometrium from the 10th day of menstrual cycle by regular vaginal ultrasound, combined with urine $\mathrm{LH}$, serum $\mathrm{LH}, \mathrm{E}_{2}$ and progesterone measurements until ovulation. One or two warmed blastocysts were transferred 5 days after ovulation.

In the hormone replacement treatment FET cycles (HRT-FET), women received escalating doses of estradiol valerate $(4 \mathrm{mg}$ daily since cycle day 3 for 6 days, 6 mg daily for 6 days). Endometrial thickness and pattern were monitored by vaginal ultrasound, and when the 
endometrial thickness was $\geq 7 \mathrm{~mm}$, progesterone $(60 \mathrm{mg}$ daily) was administrated. One or two blastocysts were transferred 5 days after progesterone administration.

In the ovulation-induction cycle FET (OI-FET), drugs such as letrozole were given to promote the development of the ovarian follicles from the 3rd day of cycle to the 7th day. Endometrium and follicle were monitored by vaginal ultrasound from the 10th day of the cycle, and 37.5 IU - 75 IU HMG was administrated if necessary. When the largest follicle exceeded $18 \mathrm{~mm}, 10,000$ IU of hCG was injected to trigger ovulation. One or two blastocysts were transferred 5 days after ovulation.

As for the GnRH-a + HRT FET protocol, GnRH-a $3.75 \mathrm{mg}$ was injected on the 2nd or the 3th day of the menstrual cycle. After 14 days GnRH-a injection, the estradiol valerate was administrated according to the HRT-FET protocol. Estradiol valerate was commenced orally with a step-up from $4 \mathrm{mg}$ daily for 6 days, to $6 \mathrm{mg}$ daily for 6 days. If the endometrial thickness was $\geq 7 \mathrm{~mm}$, progesterone (60 mg daily) was administrated.

In all FET cycles, one or two cryopreserved blastocysts were warmed and transferred. The primary outcome was clinical pregnancy, and the secondary observation index was biochemical pregnancy. Clinical pregnancy was confirmed by ultrasound visualization of a gestational sac 4-5 weeks after blastocyst transfer. Biochemical pregnancy was determined with a serum hCG $>10$ IU/L 12-14 days after blastocyst transfer. Luteal support would last for 1 month at least after clinical pregnancy confirmation.

\section{Statistical analysis}

All data analyses were conducted with SPSS software version 23.0 (IBM, New York, USA). Continuous data were showed as the mean \pm standard deviation (SD), and subjected to a Student's t-test. Categorical data were described as a frequency and percentages and analyzed using a Chi-square test. The relationship between pregnancy outcomes and morphological variables were analyzed by multivariate logistic regression analysis. $P<0.05$ was set as statistically significant.

\section{Results}

In total, 1154 frozen-warmed blastocysts transfer cycles were included, involving 725 single-blastocyst transfer cycles and 429 two-blastocysts transfer cycles. In total, 1583 viable blastocysts were transferred, and 568 blastocysts implanted. The implantation rate was $35.88 \%$ (568/1583) and the clinical pregnancy rate was $37.6 \%(434 / 1154)$. The majority of blastocysts were of expansion grade 4 (57.30\%) and 3 (31.65\%). Characteristics of patients and blastocyst morphology were summarized in Table 1.

According to clinical pregnancy outcome, 1154 frozen blastocyst transfer cycles were divided into two groups:
Table 1 Characteristic of patients and blastocyst morphology $(N=1154)$

\begin{tabular}{|c|c|}
\hline Characteristic & Mean \pm SD / N / \% \\
\hline Female age(years) & $31.39 \pm 5.48$ \\
\hline No. of early cycles & $2.65 \pm 1.06$ \\
\hline Duration of infertility (years) & $5.68 \pm 3.13$ \\
\hline Endometrial thickness (mm) & $9.42 \pm 1.65$ \\
\hline Expansion of blastocyst & $3.59 \pm 0.75$ \\
\hline degree 1 & $21(1.33 \%)$ \\
\hline degree 2 & $87(5.50 \%)$ \\
\hline degree 3 & $501(31.65 \%)$ \\
\hline degree 4 & $907(57.30 \%)$ \\
\hline degree 5 & $52(3.28 \%)$ \\
\hline degree 6 & $15(0.95 \%)$ \\
\hline Cycles of clinical pregnancy & $434(37.6 \%)$ \\
\hline Cycles of non-pregnancy & $720(62.4 \%)$ \\
\hline Cycles with single blastocyst transfer & $725(62.8 \%)$ \\
\hline Cycles with two blastocysts transfer & $429(37.2 \%)$ \\
\hline Total No. of blastocysts transferred & 1583 \\
\hline Cycles with one embryo sac & 300 \\
\hline Cycles with two embryo sacs & 134 \\
\hline Total No. of blastocyst implanted & 568 \\
\hline Implantation rate & $35.88 \%$ \\
\hline \multicolumn{2}{|l|}{ Type of infertility } \\
\hline Primary infertility & $518(44.9 \%)$ \\
\hline Secondary infertility & $636(55.1 \%)$ \\
\hline \multicolumn{2}{|l|}{ Protocol for endometrial preparation } \\
\hline GnRH-a + HRT-FET & $82(7.1 \%)$ \\
\hline HRT-FET & $627(54.3 \%)$ \\
\hline Ol-FET & $51(4.4 \%)$ \\
\hline NC-FET & 394(34.1) \\
\hline
\end{tabular}

Categorical variable is present as $\mathrm{n}(\%)$, and continuous variable is present as Mean \pm SD. NC Natural cycle, HRT Hormone replacement treatment, $\mathrm{Ol}$ ovulation-induction

clinical pregnancy group $(n=434)$ and non-clinical pregnancy group $(n=720)$. There were no significantly differences between the two groups concerning the duration of infertility, No. of early cycles, protocol for endometrial preparation, the pattern of endometrium, No. of blastocyst transferred, distribution of ICM and TE. The patients who achieved clinical pregnancy were younger $(30.68 \pm 5.25$ vs. $31.39 \pm 5.70, P=0.034)$, and had a significantly thicker endometrium $(9.65 \pm 1.63$ vs. $9.28 \pm 1.64, P=0.000)$ and a significantly higher degree of blastocyst expansion ( $3.69 \pm 0.68$ vs. $3.53 \pm 0.78$, $P=0.000)$ compared with those with non-clinical pregnancy. In the three blastocyst morphology parameters, the degree of blastocoele expansion was the most relevant factor for clinical pregnancy (Table 2). 
Table 2 Characteristics of patient and blastocyst morphology divided by clinical pregnancy

\begin{tabular}{|c|c|c|c|}
\hline Variable & Clinical pregnancy $(n=434)$ & Non-clinical pregnancy $(n=720)$ & $P$-value \\
\hline Female age(y) & $30.68 \pm 5.25$ & $31.39 \pm 5.70$ & 0.034 \\
\hline Duration of infertility(y) & $5.77 \pm 2.94$ & $5.63 \pm 3.24$ & 0.731 \\
\hline \multicolumn{4}{|l|}{ Type of infertility } \\
\hline Primary infertility & $215(49.5 \%)$ & $303(42.1 \%)$ & \\
\hline Secondary infertility & $219(50.5 \%)$ & $417(57.9 \%)$ & 0.140 \\
\hline No. of early cycles & $2.66 \pm 1.04$ & $2.64 \pm 1.07$ & 0.794 \\
\hline \multicolumn{4}{|l|}{ Protocol of FET } \\
\hline NC-FET & 154(35.5\%) & $240(33.3 \%)$ & \\
\hline HRT-FET & $220(50.7 \%)$ & $407(56.5 \%)$ & \\
\hline Ol-FET & $20(4.6 \%)$ & $31(4.3 \%)$ & \\
\hline GnRH-a + HRT-FET & $40(9.2 \%)$ & $42(5.8 \%)$ & 0.090 \\
\hline No. of blastocyst transferred & $1.34 \pm 0.48$ & $1.39 \pm 0.49$ & 0.121 \\
\hline Endometrial thickness (mm) & $9.65 \pm 1.63$ & $9.28 \pm 1.64$ & 0.000 \\
\hline \multicolumn{4}{|l|}{ Endometrial pattern } \\
\hline A & 123(28.3\%) & $187(26.0 \%)$ & \\
\hline B & $280(64.5 \%)$ & $479(66.5 \%)$ & \\
\hline C & $31(7.1 \%)$ & $54(7.5 \%)$ & 0.677 \\
\hline Blastocoele expansion & $3.69 \pm 0.68$ & $3.53 \pm 0.78$ & 0.000 \\
\hline 1 & $5(0.9 \%)^{a, b, c, d}$ & $16(1.6 \%)^{a, b, c, d}$ & \\
\hline 2 & $21(3.6 \%)^{c, d}$ & $66(6.6 \%)^{c, d}$ & \\
\hline 3 & $156(26.8 \%)^{b, d}$ & $345(34.5 \%)^{b, d}$ & \\
\hline 4 & $373(64.0 \%)^{a}$ & $534(53.4 \%)^{a}$ & \\
\hline 5 & $24(4.1 \%)^{a, b, c, d}$ & $28(2.8 \%)^{a, b, c, d}$ & \\
\hline 6 & $4(0.7 \%)^{a, b, c, d}$ & $11(1.1 \%)^{a, b, b, d}$ & \\
\hline \multicolumn{4}{|l|}{ ICM grade } \\
\hline A & $83(14.2 \%)$ & $127(12.7 \%)$ & \\
\hline B & $445(76.3 \%)$ & $785(78.5 \%)$ & \\
\hline C & $55(9.4 \%)$ & $88(8.8 \%)$ & 0.594 \\
\hline \multicolumn{4}{|l|}{ TE grade } \\
\hline A & $96(16.5 \%)$ & $148(14.8 \%)$ & \\
\hline B & $416(71.4 \%)$ & 694(69.4\%) & \\
\hline C & $71(12.2 \%)$ & $158(15.8 \%)$ & 0.122 \\
\hline
\end{tabular}

Variables were present as mean $\pm S D, \%$ or $n$

ICM inner cell mass, TE trophectoderm

For blastocoele expansion evaluation, groups (in the same columns) with the same letters have no significantly difference

According to biochemical pregnancy, 1154 cycles were divided into biochemical pregnancy group $(n=599)$ and non-biochemical pregnancy group $(n=555)$. Like the results of clinical pregnancy evaluation, there were no significantly differences in the duration of infertility, the type of infertility, number of early cycles, protocol of FET, No. of blastocyst transferred, endometrial pattern, ICM grade between the two groups $(P>0.05)$. Women in biochemical pregnancy group were younger than those without biochemical pregnancy $(31.04 \pm 5.31$ vs. $31.80 \pm 5.77, P=0.019)$, and had a significantly thicker endometrium compared with women without biochemical pregnancy $(9.52 \pm 1.61$ vs. $9.31 \pm 1.68, P=0.032)$. When the blastocyst parameters were evaluated, the mean EH stage of blastocyst transferred was higher in the women with biochemical pregnancy than those without biochemical pregnancy $(3.66 \pm 0.69$ vs. $3.51 \pm 0.79$, $P=0.000)$. The biochemical pregnancy group had a larger proportion of blastocyst with TE grade B (71.9\%) and smaller proportion of TE grade C (12.4\%) compared with women without biochemical pregnancy (Additional file 1 Table S1). 
With logistic regression analysis, we assessed the correlation between clinical pregnancy/biochemical pregnancy and blastocyst morphology parameters and cycle characteristics (For biochemical pregnancy, see Additional file 2: Table S2). For clinical pregnancy, the endometrial thickness and the blastocoele expansion degree were significantly associated. Although the difference in the endometrial thickness was significant, the absolute difference in endometrial thickness between groups was less than $1 \mathrm{~mm}$ and was considered to be of no clinical significance. The clinical pregnancy rate increased from $23.8 \%$ with blastocoele expansion degree 1 to $46.2 \%$ with blastocoele expansion degree 5. The other characteristics including female age, type of infertility, No. of early cycles, protocol of FET, endometrial pattern, No. of blastocyst transferred, ICM grade and TE grade, were not significantly relevant to the clinical pregnancy in this sample (Table 3). The clinical pregnancy rates of subgroups with different blastocoele expansion degree (degree 1-6) were 23.8, 24.1, 31.1, 41.1, 46.2 and $26.7 \%$, respectively, and these values were obviously different $(P=0.000)$ (Additional file 3 Table S3). For biochemical pregnancy, besides the endometrial thickness and the blastocoele expansion degree, the blastocyst TE was associated with the outcome $(P=0.035)$.
The biochemical pregnancy rates were 33.3, 40.2, 46.7, 56.7, 53.8, and $46.7 \%$ in the subgroups with blastocoele expansion degree 1-6, respectively, and there were significant differences between subgroups $(P=0.004)$ (Additional file 3 Table S3). When the blastocyst TE grade was evaluated, we found the TE grade $\mathrm{B}$ have the highest biochemical pregnancy rate (53.4\%), followed by subgroup with grade A (53.3\%), and grade $C(44.5 \%)$, and there was significantly difference between subgroup with TE grade $B$ and $C$ $(P=0.046)$ (Additional file 3 Table S3).

The study assessed the clinical pregnancy and biochemical pregnancy by combined blastocyst morphology. The results showed the clinical pregnancy and biochemical pregnancy rates were associated with the blastocoele expansion degree when they have the same ICM and TE grade, and transferring blastocyst with blastocoele expansion degree 4 and 5 had achieved a better clinical outcome. Whereas with the same blastocoele expansion degree, there was no significantly difference in clinical pregnancy rate and biochemical pregnancy rate between subgroups with different combined ICM grade and TE grade $(P>0.05)$ (Additional file 4 Table S4). For blastocysts with expansion 3-5, TE grade had a more effect on the biochemical pregnancy with positive HCG, clinical pregnancy (Additional file 4 Table S4).

Table 3 Logistic model for predicting clinical pregnancy

\begin{tabular}{|c|c|c|c|c|c|c|c|c|}
\hline \multirow[t]{2}{*}{ Variable } & \multirow[t]{2}{*}{ B } & \multirow[t]{2}{*}{ S.E. } & \multirow[t]{2}{*}{ Wald } & \multirow[t]{2}{*}{$d f$} & \multirow[t]{2}{*}{ Sig. } & \multirow[t]{2}{*}{$\operatorname{Exp}(B)$} & \multicolumn{2}{|c|}{ 95\% Cl for $\operatorname{Exp}(B)$} \\
\hline & & & & & & & Lower & Upper \\
\hline Age & -0.021 & 0.012 & 2.963 & 1 & 0.085 & 0.979 & 0.957 & 1.003 \\
\hline Type of infertility & -0.242 & 0.128 & 3.586 & 1 & 0.058 & 0.785 & 0.611 & 1.009 \\
\hline No. of early cycles & 0.020 & 0.059 & 0.119 & 1 & 0.731 & 1.021 & 0.908 & 1.147 \\
\hline Protocol of FET & / & / & 5.072 & 3 & 0.167 & / & / & / \\
\hline Protocol of FET(1) & 0.481 & 0.252 & 3.654 & 1 & 0.056 & 1.618 & 0.988 & 2.651 \\
\hline Protocol of FET(2) & -0.063 & 0.140 & 0.203 & 1 & 0.652 & 0.939 & 0.713 & 1.236 \\
\hline Protocol of FET(3) & 0.073 & 0.317 & 0.053 & 1 & 0.817 & 1.076 & 0.578 & 2.004 \\
\hline Endometrial thickness & 0.134 & 0.039 & 11.904 & 1 & 0.001 & 1.143 & 1.059 & 1.233 \\
\hline Endometrial pattern & / & / & 0.683 & 2 & 0.711 & / & / & / \\
\hline Endometrial pattern(1) & 0.152 & 0.261 & 0.340 & 1 & 0.560 & 1.164 & 0.698 & 1.942 \\
\hline Endometrial pattern(2) & 0.043 & 0.244 & 0.031 & 1 & 0.859 & 1.044 & 0.648 & 1.683 \\
\hline No. of blastocyst transferred & -0.189 & 0.132 & 2.040 & 1 & 0.153 & 0.828 & 0.639 & 1.073 \\
\hline Blastocoele expansion & 0.262 & 0.091 & 8.253 & 1 & 0.004 & 1.300 & 1.087 & 1.555 \\
\hline ICM grade & / & / & 0.315 & 2 & 0.854 & / & / & / \\
\hline ICM grade(1) & 0.158 & 0.300 & 0.279 & 1 & 0.597 & 1.172 & 0.651 & 2.108 \\
\hline ICM grade(2) & 0.134 & 0.253 & 0.282 & 1 & 0.595 & 1.144 & 0.697 & 1.878 \\
\hline TE grade & / & / & 2.682 & 2 & 0.262 & / & / & / \\
\hline TE grade(1) & 0.324 & 0.246 & 1.733 & 1 & 0.188 & 1.383 & 0.853 & 2.242 \\
\hline TE grade(2) & 0.315 & 0.195 & 2.608 & 1 & 0.106 & 1.371 & 0.935 & 2.011 \\
\hline Constant & -2.165 & 0.837 & 6.682 & 1 & 0.010 & 0.115 & / & / \\
\hline
\end{tabular}




\section{Discussion}

The present study showed that blastocoele expansion degree was significantly associated with the clinical pregnancy and biochemical pregnancy rates after one or two frozen blastocysts transfer. It has been reported that blastocoel expansion is important for clinical outcomes. A study by Goto et al. [11] investigated 1488 FET cycles with single blastocyst transfer and found that it was the blastocoele expansion degree rather than ICM/ TE that significantly affected pregnancy outcomes. Ahlstrom et al. [7] and Thompson et al. [9] reported that the blastocyst expansion degree was a significant and independent predictor of live birth rates of fresh single blastocyst transfer cycle. Recently, one study by Sun YP et al. [5] confirmed that the blastocoele expansion degree had a better predictive value than ICM or TE grade in predicting live birth after both fresh and vitrified/ warmed single blastocyst transfer cycles.

As stated in previous researches, blastocoele expansion stage has a lot of significant functions in the early stages of blastocyst implantation. These functions involve hatching from the zona pellucida, hCG-mediated signaling, adhesion and invasion of the endometrium, and dialogue with the mother [22-27], which are essential for a successful pregnancy as well as prevention of abortion. What have been stated above may explain our results that blastocoele expansion degree has more predictive information than TE and ICM stage.

Certainly, there have been many studies indicated the predictive value of TE or ICM stage. The possible explanations for such different conclusions may be as follows: Firstly, the distribution of different grades blastocysts is diverse between studies, which may explain the obvious inconsistency. For example, some studies evaluated the effect of blastocyst parameters on the clinical outcome after selective single blastocyst transfer in fresh cycles or in FET cycles. Most of blastocysts transferred have good or even excellent quality, and a very small part of blastocysts has $\mathrm{C}$ grade for ICM or TE. But, the data in the present study were derived from a retrospective clinical trial in which one or two blastocysts were transferred regardless of the quality of blastocyst, thus a number of blastocysts with blastocoele expansion degree 1-2 and ICM and TE with grade $C$ were included. Secondly, in spite of the fact that these studies applied the same blastocyst grading system with written definitions and common training courses, the discursive concept of the ICM and TE morphology was an intrinsic defect in the blastocyst grading system proposed by Gardner and Schoolcraft [6], and subjective assessment was inevitable when grading the blastocyst.

In the present study, we found that majority of blastocysts had blastocoele expansion 3-5 and the grade of ICM and TE were highly correlated: ICM and TE A and
$B$ grade were more likely correlated with TE and ICM A and $\mathrm{B}$ grade, for example, $\mathrm{AA}, \mathrm{AB}, \mathrm{BA}, \mathrm{BB}$, or $\mathrm{CC}$ grades appeared more likely than other combinations (i.e. AC, CA grades). These observations showed interdependence between ICM and TE during the blastocyst developmental phase. As discussed by Ahlstrom et al. [7], the blastocoele expansion is relevant to the cell numbers and cohesive property of the TE, preventing outflow of blastocyst fluid and sodium ions. Therefore, a good TE grade may manifest that the blastocyst effectively pumps ions into the cavity and induces the permeability accumulation of intracellular water, leading to blastocoele expansion [7]. That is to say, by definition, functional TE cells are required to a fully expanded blastocyst, and this function may be more relevant to the molecular quality of TE cells than its number and cohesion. A better understanding of the correlation between these three morphological parameters helps to choose the best blastocyst for transfer.

Some studies suggested that blastocyst in earlier development stage and with lower blastocoele expansion degree have higher survival rate, implantation rate and live birth rates, which contradict to our findings [20, 28-31]. The method of vitrification used should be considered. Some studies have not used artificial shrinking of the blastocoele cavity to obtain higher-grade blastocysts. In these studies, the blastocoele fluid was regarded to be a barrier to dehydration and pervasion of cryoprotectant along with a negative impact on the blastocyst survival and implantation. Whereas, once blastocyst received artificial shrinkage, blastocysts with higher expansion degree owned the same or a higher survival rate, implantation rate and live birth rate compared to blastocysts with lower developmental degree [32-34]. Small blastomeres with larger surface area to volume ratio have been shown to be less sensitive to osmotic pressure and damage. The reason is that cryoprotectant penetrates into smaller cells faster [35]. Thus, higher expansion degree blastocysts with smaller balstomeres would most likely be more resistant to vitrification and high concentrations of cryoprotectants.

The strength of the present study was its large sample size of 1154 transfer cycles, and total 1583 warmed blastocysts transferred. Besides, different with previous studies which included good blastocyst, this study assessed all kinds of viable blastocysts $>3 \mathrm{CC}$ and did not limit to good quality blastocyst $(\geq 3 \mathrm{BB})$. And our results found that ICM $C$ grade or TE $C$ grade blastocyst with higher blastocoele expansion degree also has optimistic clinical results. In addition, the present study firstly assessed FET cycles with single blastocyst or two blastocysts transfer, and cycles in which blastocysts transferred were all implanted or all not implanted were included. At present, selective single embryo/blastocyst transfer 
and blastocyst culture were not routine practices. When there still have surplus embryos after fresh embryo transfer, these embryos, including not top-quality embryos, were proposed to be cultured to blastocyst stage.

Certainly, this study has a few limitations. The first one was its retrospective design, and some bias was inevitable. Secondly, this study did not observe other clinical outcomes, such as ongoing pregnancy rate, abortion rate, and live birth rate. As the follow-up has not been completed in some cases by now, so it is hard to compare these outcomes. Besides, live birth was affected by too many factors except for blastocyst quality. So, the present included the biochemical pregnancy and clinical pregnancy, which were surely associated with blastocyst quality. Thirdly, the present study only evaluated the blastocoele expansion degree after embryo thawing, and did not evaluate the expansion degree before vitrification. Recent study showed that the degree of blastocyst expansion after vitrification/thawing was almost the same as that before vitrification [1]. In spite of these shortcomings, the present study analyzed sis the parameters of blastocysts with a valuable summary.

\section{Conclusion}

The blastocyst expansion is a significant parameter for predicting clinical success pregnancy after frozen-thawed blastocysts transfer and should be given the priority when selecting frozen blastocyst for transfer.

\section{Additional files}

Additional file 1: Table S1. Characteristics of patient and blastocyst morphology divided by biochemical pregnancy. (DOCX 88 kb)

Additional file 2: Table S2. Logistic model for predicting biochemical pregnancy. (DOCX $109 \mathrm{~kb}$ )

Additional file 3: Table S3.Comparison of clinical pregnancy rate and biochemical pregnancy rate between different subgroups according to blastocoele expansion degree and TE grade. (DOCX $70 \mathrm{~kb}$ )

Additional file 4: Table S4. Comparison of outcomes between different subgroups divided according by blastocyst ICM/TE grade with the same expansion degree. (DOCX $81 \mathrm{~kb})$

\section{Abbreviations}

ART: assisted reproductive technology; FET: frozen-thawing embryo transfer; ICSI: intracytoplasmic sperm injection; IUA: intrauterine adhesion; IVF: in vitro fertilization; PGD/PGS: preimplantation genetic diagnosis/screening

\section{Acknowledgments}

Not applicable.

\section{Funding}

This project was supported by the National Natural Science Foundation of China (Grant No. 81401269), and the class General Financial Grant F from the China Postdoctoral Science Foundation (Grant No. 2017 M620360).

\section{Availability of data and materials}

The datasets supporting the conclusions of this article are included within the article and its additional file.

\section{Authors' contributions}

ZJ contributed to the study design and acquisition of data, article drafting and revising as well as organization of the final approval of the version to be published. YY contributed substantially to the interpretation of the data and helped with drafting and revision of the article. SL and LY contributed to conception and design, acquisition of data and analysis and interpretation of data. HX contributed significantly to the acquisition, analysis and interpretation of the data.

Ethics approval and consent to participate

Not applicable

\section{Consent for publication}

Not applicable.

\section{Competing interests}

The authors declare that they have no competing interests.

\section{Publisher's Note}

Springer Nature remains neutral with regard to jurisdictional claims in published maps and institutional affiliations.

\section{Author details}

${ }^{1}$ Reproductive Medicine Center, Xiangya Hospital, Central South University, 87 Xiangya Road, Changsha City, Hunan Province, People's Republic of China. ${ }^{2}$ Center for Molecular Medicine, Xiangya Hospital, Central South University, Hunan, People's Republic of China.

Received: 19 September 2018 Accepted: 9 January 2019

Published online: 23 January 2019

\section{References}

1. Cruz JR, Dubey AK, Patel J, Peak D, Hartog B, Gindoff PR. Is blastocyst transfer useful as an alternative treatment for patients with multiple in vitro fertilization failures? Fertil Steril. 1999;72:218-20.

2. Papanikolaou EG, Kolibianakis EM, Tournaye $H$, Venetis CA, Fatemi $H$, Tarlatzis B, et al. Live birth rates after transfer of equal number of blastocyst or cleavage-stage embryos in IVF. A Syst Rev Meta- Analysis Hum Reprod. 2008:23:91-9.

3. Zech $\mathrm{NH}$, Lejeune $\mathrm{B}$, Puissant $\mathrm{F}$, Vanderzwalmen $\mathrm{S}$, Zech $\mathrm{H}$, Vanderzwalmen $P$. Prospective evaluation of the optimal time for selecting a single embryo for transfer: day 3 versus day 5. Fertil Steril. 2007;88:244-6.

4. Zhu D, Zhang J, Cao S, Heng BC, Huang M, Ling $X$, et al. Vitrified- warmed blastocyst transfer cycles yield higher pregnancy and implantation rates compared with fresh blastocyst transfer cycles-time for a new embryo transfer strategy? Fertil Steril. 2011;95:1691-5.

5. Du QY, Wang EY, Huang Y, Guo XY, Xiong YJ, Yu YP, et al. Blastocoele expansion degree predicts live birth after single blastocyst transfer for fresh and vitrified/warmed single blastocyst transfer cycles. Fertil Steril. 2016;105:910-9.

6. Gardner DK, Schoolcraft WB. In vitro culture of human blastocysts. In: Jansen $R$, Mortimer $D$, editors. Towards reproductive certainty: fertility and genetics beyond. Carnforth: Parthenon Publishing; 1999. p. 378-88.

7. Ahlstrom A, Westin C, Reismer E, Wikland M, Hardarson T. Trophectoderm morphology: an important parameter for predicting live birth after single blastocyst transfer. Hum Reprod. 2011;26:3289-96.

8. Hill MJ, Richter KS, Heitmann RJ, Graham JR, Tucker MJ, DeCherney AH, et al. Trophectoderm grade predicts outcomes of single-blastocyst transfers. Fertil Steril. 2013:99:1283-9.e1.

9. Thompson SM, Onwubalili N, Brown K, Jindal SK, McGovern PG. Blastocyst expansion score and trophectoderm morphology strongly predict successful clinical pregnancy and live birth following elective single embryo blastocyst transfer (eSET): a national study. J Assist Reprod Genet. 2013;30:1577-81.

10. Chen X, Zhang J, Wu X, Cao S, Zhou L, Wang Y, et al. Trophectoderm morphology predicts outcomes of pregnancy in vitrified- warmed singleblastocyst transfer cycles in a Chinese population. J Assist Reprod Genet. 2014;31:1475-81.

11. Goto S, Kadowaki T, Tanaka S, Hashimoto H, Kokeguchi S, Shiotani M. Prediction of pregnancy rate by blastocyst morphological score and age, based on 1488 single frozen-thawed blastocyst transfer cycles. Fertil Steril. 2011;95:948-52. 
12. Richter KS, Harris DC, Daneshmand ST, Shapiro BS. Quantitative grading of a human blastocyst: optimal inner cell mass size and shape. Fertil Steril. 2001;76:1157-67.

13. Van den Abbeel E, Balaban B, Ziebe S, Lundin K, Cuesta MJ, Klein BM, et al. Association between blastocyst morphology and outcome of singleblastocyst transfer. Reprod BioMed Online. 2013;27:353-61.

14. Ahlstrom A, Westin C, Wikland M, Hardarson T. Prediction of live birth in frozen- thawed single blastocyst transfer cycles by pre-freeze and post-thaw morphology. Hum Reprod. 2013;28:1199-209.

15. Zhu L, Che HS, Xiao L, Li YP. Uterine peristalsis before embryo transfer affects the chance of clinical pregnancy in fresh and frozen-thawed embryo transfer cycles. Hum Reprod. 2014;29:1238-43.

16. Zhao J, Zhang Q, Wang YG, Li YP. Endometrial pattern, thickness and growth in predicting pregnancy outcome following 3319 IVF cycle. Reprod BioMed Online. 2014;29:291-8.

17. Zhao J, Zhang Q, Li YP. The effect of endometrial thickness and pattern measured by ultrasonography on pregnancy outcomes during IVF-ET cycles. Reprod Biol Endocrin. 2012;10:100.

18. Zhu L, Li YP, Xu AZ. Influence of controlled ovarian hyperstimulation on uterine peristalsis in fertile women. Hum Reprod. 2012;27:2684-9.

19. Lane M, Schoolcraft WB, Gardner DK. Vitrification of mouse and human blastocysts using a novel cryoloop container- less technique. Fertil Steril. 1999;72:1073-8.

20. Mukaida T, Nakamura S, Tomiyama T, Wada S, Oka C, Kasai M, Takahashi K. Vitrification of human blastocysts using cryoloops: clinical outcome of 223 cycles. Hum Reprod. 2003;18:384-91.

21. Mukaida T, Takahashi K, Kasai M. Blastocyst cryopreservation: ultrarapid vitrification using cryoloop technique. Reprod BioMed Online. 2003;6:221-5.

22. Licht $P$, Russu V, Lehmeyer S, Wildt L. Molecular aspects of direct $\mathrm{LH} / \mathrm{hCG}$ effects on human endometrium - lessons from intrauterine microdialysis in the human female in vivo. Reprod Biol. 2001;1:10-9.

23. Licht $P$, Russu V, Wildt L. On the role of human chorionic gonadotropin ( $\mathrm{hCG}$ ) in the embryo-endometrial microenvironment: implications for differentiation and implantation. Semin Reprod Med. 2001;19:37-47.

24. Tasampalas M, Gridelet V, Berndt S, Foidart JM, Geenen V, Perrier d' Hauterive S. Human chorionic gonadotropin: a hormone with immunological and angiogenic properties. J Reprod Immunol. 2010;85:93-8.

25. Jones GM, Cram DS, Song B, Kokkali G, Pantos K, Trounson AO. Novel strategy with potential to identify developmentally competent IVF blastocysts. Hum Reprod. 2008;23:1748-59.

26. Alfarawati S, Fragouli E, Colls P, Stevens J, Gutierrez-Mateo C, Schoolcraft WB, Katz-Jaffe MG, Wells D. The relationship between blastocyst morphology, chromosomal abnormality, and embryo gender. Fertil Steril. 2011;95:520-4

27. Parks JC, McCallie BR, Janesch AM, Schoolcraft WB, Katz-Jaffe MG. Blastocyst gene expression correlates with implantation potential. Fertil Steril. 2011;95:1367-72.

28. Cho HJ, Son WY, Yoon SH, Lee SW, Lim JH. An improved protocol for dilution of cryoprotectants from vitrified human blastocysts. Hum Reprod. 2002;17:2419-22.

29. Van den Abbeel E, Camus M, Verheyen G, Van Waesberghe L, Devroey P, Van Steirteghem A. Slow controlled- rate freezing of sequentially cultured human blastocysts: an evaluation of two freezing strategies. Hum Reprod. 2005;20:2939-45.

30. Zech NH, Lejeune B, Zech $\mathrm{H}$, Vanderzwalmen P. Vitrification of hatching and hatched human blastocysts: effect of an opening in the zona pellucida before vitrification. Reprod BioMed Online. 2005:11:355-61.

31. Ebner T, Vanderzwalmen $P$, Shebl O, Urdl W, Moser M, Zech NH, Tews G. Morphology of vitrified/ warmed day-5 embryos predicts rates of implantation, pregnancy and live birth. Reprod BioMed Online. 2009;19:72-8.

32. Son WY, Yoon SH, Yoon HJ, Lee SM, Lin JH. Pregnancy outcome following transfer of human blastocysts vitrified on electron microscopy grids after induced collapse of the blastocoele. Hum Reprod. 2003;18:137-9.

33. Mukaida T, Oka C, Goto T, Takahashi K. Artificial shrinkage of blastocoeles using either a micro-needle or a laser pulse prior to the cooling steps of vitrification improves survival rate and pregnancy outcome of vitrified human blastocysts. Hum Reprod. 2006;21:3246-52.

34. Wikland M, Hardarson T, Hillensjo T, Westin C, Westlander G, Wood M, et al. Obstetric outcomes after transfer of vitrified blastocysts. Hum Reprod. 2010; 25:1699-707.

35. Tachikawa S, Otoi T, Kondo S, Machida T, Kasai M. Successful vitrification of bovine blastocysts, derived by in vitro maturation and fertilization. Mol Reprod Dev. 1993;34:266-71.

Ready to submit your research? Choose BMC and benefit from:

- fast, convenient online submission

- thorough peer review by experienced researchers in your field

- rapid publication on acceptance

- support for research data, including large and complex data types

- gold Open Access which fosters wider collaboration and increased citations

- maximum visibility for your research: over $100 \mathrm{M}$ website views per year

At BMC, research is always in progress.

Learn more biomedcentral.com/submissions 